\title{
Truels, OR SURVIVAL OF THE WeAKEST
}

\author{
By Pau Amengual and Raúl Toral
}

W

E’RE ALL FAMILIAR WITH THE CONCEPT OF DUELS.

THEY CAN BE DEADLY, SUCH AS THOSE FOUGHT BE-

TWEEN TWO SUITORS VYING FOR A LADY'S AFFECTIONS OR

SHERIFFS AND OUTLAWS BATTLING IT OUT IN THE AMERICAN

Old West, or they can end in both parties enjoying a beer, with the loser buying a round after an intense game of darts. A duel is simply a game with two players, each having a probability of winning that game and each having an intrinsic marksmanship, or ability, associated with his or her performance. Of course, the worst player-the one with the worst marksmanship-might have an advantage such as extra bullets or extra points, but in a normal unbiased situation, common sense tells us that the higher the marksmanship, the higher the probability of winning the duel. The mathematical treatment of this game confirms our simple expectations. If more than two players participate, a series of duels might be needed to determine the absolute winner. This is the case in sports tournaments, which pair teams in all possible ways until the ultimate winner emerges.

A truel is a generalization of a duel involving three players and slightly different rules. First, we assume that a player's marksmanship doesn't depend on his or her opponent - the probability that a person kills an opponent in a romantic duel, for example, won't depend on his opponent's identity. Second, truelists shoot sequentially, one after another-if a truelist misses a shot, then it's another truelist's turn. Third, each truelist has the right to choose which person to aim at. In a random truel,

1. One of the remaining truelists is chosen at random.

2. The chosen truelist selects an opponent and, with a certain probability (marksmanship), eliminates that opponent from the game.

3. Whatever the result, steps 1 and 2 repeat until there is only one survivor, the winner.

Truels produce a paradoxical result: the player with the highest marksmanship doesn't necessarily win. ${ }^{1,2}$ It's an example of a situation in which "the fittest does not necessarily survive." We all know of cases where the best person didn't get the job or become manager; truels present a framework in which this counterintuitive outcome can be rigorously derived. These games first appeared in the $1940 \mathrm{~s},{ }^{4}$ but the term truel wasn't coined until the 1960s. In this article, we'll investigate truels in more detail.

\section{All about Truels}

Truels are interesting from a pedagogical viewpoint because they illustrate in a particularly clear way some of the concepts of game theory. In particular, consider the Nash equilibrium-the best action players can take to optimize their probability of winning the game. The action here corresponds to the strategy of choosing an opponent. Intuition tells us that your optimum strategy given the rules is to shoot at the best remaining player. This "best opponent" strategy explains the paradoxical result because it implies that the best player is the target of the other two, thus decreasing that player's probability of winning.

Previous researchers have confirmed this intuitive explanation via game theory. ${ }^{5}$ We've revisited ${ }^{6}$ such analysis from the viewpoint of stochastic processes using discrete-time Markov chains ${ }^{7}$ with three absorbing states. The players still in the game determine the chain states; the absorbing states correspond to the state in which only one player remains. With this technique, we can calculate the probability of the system ending in one of three absorbing states-that is, each player's probability of winning. This viewpoint, besides reproducing the main paradoxical result, provides a framework for analyzing games with which some communities (such as physicists) feel more comfortable. In fact, physics has contributed to the field of paradoxical games with Spanish physicist Juan Parrondo's games. ${ }^{8}$ These games exemplify the situation in which a combination of losing games results in a winning one. They're inspired by the ratchet and pawl, a pedagogical device 
that Maryan von Smoluchovsky ${ }^{9}$ invented and Richard Feynman studied extensively ${ }^{10,11}$ to illustrate the second law of thermodynamics, which poses a limit on how much energy we can extract from a given source.

The literature has other models that also give counterintuitive results, such as the rock-scissors-paper game (in which rock beats a pair of scissors, scissors beat a sheet of paper, and paper beats rock). Researchers have applied it to population dynamics, ${ }^{12,13}$ toospecifically, to systems with three species that interact with each other and ultimately create a competitive loop. The paradoxical effect in this model is that the least competitive species might be the one with the largest population and, even when there are oscillations in a finite population, the least likely to die out. Researchers have also applied this game to a voter model, ${ }^{14}$ obtaining again a paradoxical result-namely, an initial decline in support for one candidate later leading to an upsurge in the polls for that same candidate.

Researchers have proposed several modifications of the basic truel rules, such as biasing the game by allowing the worst player to shoot first and the best a priori player to shoot last. ${ }^{15} \mathrm{Al}-$ though it isn't surprising that this "sequential rule" increases the probability of the worst player winning the game, it is surprising that, to increase the survival probability, the worst player might need to miss those shots on purpose. Another option (not considered in this article) is the simultaneous truel, in which the three players shoot at the same time.

We could also change some of the other rules-is the number of rounds finite or infinite? Will the ammunition be limited or unlimited? These slight tweaks change the probabilities of dif- ferent outcomes. ${ }^{15,16}$ Another modification is cooperative truels, ${ }^{17}$ which are characterized by the appearance of alliances-different players set a common target and improve their survival probability by forming a coalition. A quantum scheme for duels and truels appears elsewhere. ${ }^{18}$

In this article, we limit ourselves to the random and sequential truels in which players use their best possible strategy with no coalitions. We opted to modify the random truel slightly and convert it into an opinion model in which each player has a different opinion on a given topic. In each round, we randomly pick one player to try and change another player's opinion. The game ends when all players share the same opinion. If we extend the truel rules from three to $N$ players, the paradoxical results show up even more clearly, because as $N$ increases, it's more difficult for the player with the highest marksmanship to win the game.

\section{The Duels}

A standard duel has two players: a good player $(A)$ and a bad player $(B)$. Let's denote their respective marksmanships with $a$ and $b$, where $a>b$. Here, the strategy is obvious: shoot at the only other opponent. It makes no sense to lose an opportunity to eliminate the opponent by shooting into the air.

In the random duel case, in which we choose the next player to shoot randomly at each time step, an analytical study (using, for example, a Markov chain) shows that a player's probability of winning is proportional to that player's marksmanship. If $P_{A}$ (or $P_{B}$ ) is the probability of player $A$ (or $B$ ) winning the game, we have $P_{A}=a /(a+b)$ (or $\left.P_{B}=b /(a+b)\right)$. So in a random duel, the best player has the largest survival probability. The same result holds if we randomly choose the first player to shoot and then they each shoot sequentially one after the other until only one remains.

We can beat this result by letting the worst player shoot first and alternate turns afterward. In this type of sequential duel, an analysis of the resulting winning probabilities shows that only when $b>a /(1+a)$ is player $B$ 's survival probability greater than $A$ 's. Therefore, in a sequential duel, player $B$ can overcome the unfavorable situation of having lower marksmanship by being the first shooter.

\section{Truel Strategies}

If a third player enters the game, the situation is no longer simple. Each player in the truel must now consider all the possible actions that the other opponents might take and their corresponding outcomes. For concreteness, and without loss of generality, let's assume that the third player $C$ has the lowest marksmanship $c$, such that $a>b>c$.

All players in the truel share the same goal: to be the sole survivor. We can explicitly impose this through the inclusion of a payoff, a concept that corresponds to some sort of reward a player receives for achieving the goal. To maximize their payoff, players must choose strategies that maximize their survival probability. When the three players are still in the game, each one has three possible strategies: two correspond to choosing one of the two opponents, and the third strategy is to shoot in the air (or miss a shot on purpose). In this article, we only consider "pure" strategies, but it's also possible to select with a given probability one of the options; such mixed strategies aren't relevant in the case of truels, though. If one of the three players is removed from the game, we're back to a duel situation, and, as discussed earlier, the only strategy is to aim at the 
Table 1. Survival probabilities $P_{A}, P_{B}$, and $P_{C}$ of players $A, B$, and $C$ for the different strategies they adopt in a random truel.*

\begin{tabular}{lccc} 
Strategy & $\boldsymbol{P}_{\boldsymbol{A}}$ & $\boldsymbol{P}_{\boldsymbol{B}}$ & $\boldsymbol{P}_{\boldsymbol{C}}$ \\
\hline$C C B$ & 0.580 & 0.348 & 0.072 \\
\hline$C C A$ & 0.434 & 0.481 & 0.085 \\
\hline$C A B$ & 0.386 & 0.407 & 0.207 \\
\hline$C A A$ & 0.242 & 0.541 & 0.218 \\
\hline$B C B$ & 0.628 & 0.155 & 0.217 \\
\hline$B C A$ & 0.483 & 0.288 & 0.229 \\
$B A B$ & 0.435 & 0.214 & 0.351 \\
\hline$B A A$ & 0.290 & 0.348 & 0.362
\end{tabular}

*Marksmanships $\mathrm{a}=1, \mathrm{~b}=0.8$, and $\mathrm{c}=0.5$.

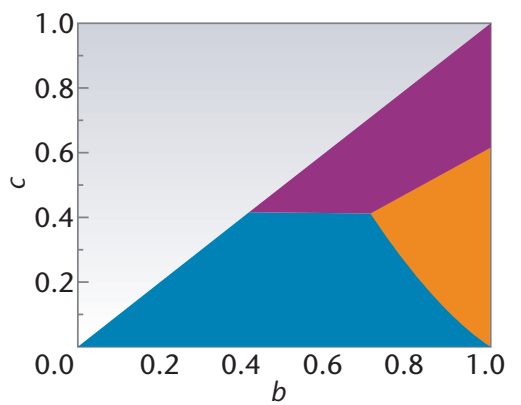

Figure 1. Random truel. The different colors (blue for player $A$, orange for player $B$, and purple for player $C$ ) indicate regions in the parameter space $(b, c)$, setting $a=1$, in which each player has the highest probability of survival.

remaining opponent. We also assume that the players' strategies are uncooperative, in the sense that alliances or pacts between them aren't allowed. The corresponding expressions for the survival probabilities appear elsewhere, ${ }^{6,15}$ but they're too cumbersome to reproduce here.

Let's first examine a random truel, in which we randomly choose a player to shoot first. If players don't use a strategy and simply shoot randomly, the winning probabilities are clearly proportional to marksmanship. But following a basic game theory premise, ${ }^{19}$ let's assume that the players are rational agents, in the sense that they adopt a strategy that's the best response to the other players' strategies. As we men- tioned earlier, this mirrors the Nash equilibrium, which we can clarify with an example. Table 1 shows the different survival—or winning-probabilities $P_{A}, P_{B}$, and $P_{C}$ of players $A, B$, and $C$, respectively, for the different strategies they adopt in a random truel. We calculated these values assuming that player $A$ has a marksmanship $a=1$ (or 100 percent effectiveness), player $B$ has $b=0.8$ (80 percent effectiveness), and player $C$ has $c=0.5$ (50 percent effectiveness). For simplicity, we don't include in this example the strategy of shooting in the air, an option that isn't relevant for the random truel.

Let's start by looking at the set of strategies $C C B$ in Table 1 . The notation means that the first player $A$ aims at $C$, the second player $B$ aims at $C$, and the third player $C$ aims at $B$. In this case, the player with the highest survival probability is $A$, with a 58 percent chance of winning, followed by player $B$ with a 34.8 percent chance, and finally player $C$, with a very low chance of 7.2 percent.

When analyzing this situation, player $C$ concludes that it's better to change strategies, so instead of aiming at $B$, sets player $A$ as a new target. This becomes row $C C A$ in the table, where $C$ 's survival probability has increased to 8.5 percent. Player $B$ looks at this revised situation and sets $A$ as a new target, which takes us to the $C A A$ row, where $P_{B}$ increases from 48.1 percent to 54.1 percent. Finally, player $A$ decides to change strategies and sets $B$ as a new target, thus taking us to $B A A$, where $P_{A}$ increases from 24.2 percent to 29.0 percent. Amazingly, if we repeat these moves starting from any strategy and using any order of player reasoning, we ultimately reach the same strategy set: $B A A$.

The $B A A$ strategy is known in the literature ${ }^{1}$ as the strongest-opponent strategy because all players aim at the opponent with the highest marksmanship: player $A$ aims at $B$, and players $B$ and $C$ aim at $A$. This is the random truel's unique Nash equilibrium point, where no player can improve his or her survival probability by changing strategy as long as the rest of the players keep theirs. This set corresponds to a local maximum of every player's survival probabilities, and it's the one that rational players will necessarily use. Remarkably, survival probability in this strategy goes in inverse order with marksmanship: player $C$ has the highest survival probability, and player $A$ has the lowest. This result is counterintuitive, in the sense that we'd naively expect player $A$ to have the highest survival probability because player $A$ has the highest marksmanship.

In the sequential truel, the worst player has the advantage of being the first to shoot. The sequence of shots is $C-B-A$, and it repeats as necessary until only one player remains. A detailed analysis ${ }^{1,6}$ reveals the existence of two different equilibrium points here, depending on the actual values of the marksmanships $a, b$, and $c$. One of the equilibrium points corresponds to the strongest-opponent strategy (as in the case of the random truel), whereas the other corresponds to the set $B A \varnothing$, meaning that player $A$ sets player $B$ as a target, player $B$ sets $A$ as a target, and $C$ decides to shoot into the air. This latter set of strategies implies that it might be better for player $C$ to fail the 
shot and let players $B$ and $A$ kill each other. Player $C$ would use the next turn to try to eliminate the remaining player and win the truel.

The player with the highest survival probability depends on the marksmanship values. In Figure 1, we indicate the regions in the parameter space $(b, c)$ after setting $a=1$, in which each player has the highest survival probability. Blue corresponds to player $A$, orange to $B$, and purple to $C$. The figure shows that it's possible for every player to be the favorite in the game, although the largest region corresponds to the best player, $A$.

For the sequential truel, the situation is somewhat different. Due to the imposed firing order $(C-B-A)$, player $A$ is the last to shoot, so the advantageous situation from high marksmanship is partially lost. Figure 2 reflects this because the region in which player $A$ is the favorite has decreased considerably compared to that of Figure 1. In fact, the a priori worst player, $C$, is the favorite in many occasions. We explained previously that the sequential truel has two equilibrium points, $B A A$ and $B A \varnothing$; the last one is the relevant one in the small purple region in Figure 2 (inside the black region).

\section{Truels as a Model of Opinion Spreading}

We can give an interesting twist to a truel (as well as eliminate some of its aggressiveness) by interpreting it as a model of opinion spreading. In this case, $A, B$, and $C$ are three opinions that people can hold on a topic. In this version of the game, players aim to convince-rather than eliminate-each other. Marksmanships $a, b$, and $c$ are now the probability of convincing another person of an opinion, and the game ends when all players share the same opinion.
In the random version, theoretical analysis shows that the only equilibrium point is in the strongestopponent strategy. The same paradoxical result still applies because the opinion with the higher marksmanship doesn't necessarily need to be the one that survives. However, as Figure 3 shows, opinion $A$ is the favorite to become the majority opinion for a larger region of values of $b$ and $c$, again setting $a=1$; it's only for a relatively small region that opinion $C$ is the favorite. The overwhelming superiority of $A$ is understandable if we recall that in this model, the total number of players remains constant throughout the game-only the opinions change. Once opinion $A$ convinces either a player with opinion $B$ or a player with opinion $C$, it's very likely that it'll eventually become the majority opinion due to its high convincing probability. Starting from the initial configuration in which the three players hold different opinions $A, B$, and $C$ and the same values of $a, b$, and $c$ as before ( $a=1, b=0.8$, and $c=0.5$ ), there's a 38.6 percent probability that opinion $A$ will become the majority opinion, and a 37.8 percent and a 23.4 percent probability that opinions $B$ and $C$ will become the majority, respectively.

\section{Distribution of Winners}

Imagine now that we set a truel competition among a population of players. The population's marksmanships are uniformly distributed in the interval $(0$, 1). We set a tournament scheme, form all possible triplets of players, and have them play a random truel (we decided on a non-lethal version in which losers can still play another game). The winner will be the player with the highest number of truels won.

On whom would you bet-for the good players with high marksmanship

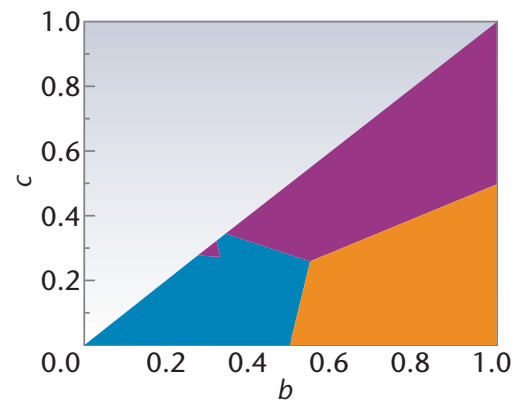

Figure 2. Sequential truel. As in Figure 1, different colors (blue for player $A$, orange for player $B$, and purple for player $C$ ) indicate regions in the parameter space $(b, c)$, setting $a=1$, in which each player has the highest probability of survival.

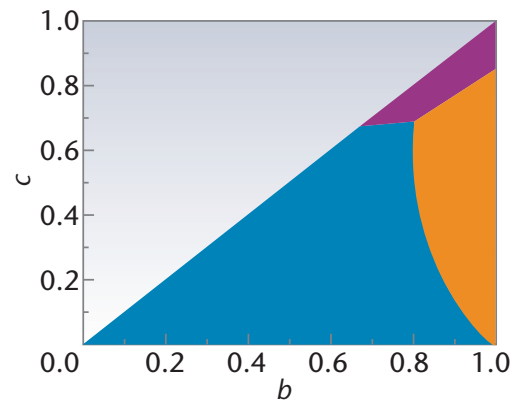

Figure 3. Random truel. The different colors (blue for player $A$, orange for player $B$, and purple for player $C$ ) indicate regions in the parameter space $(b, c)$, setting $a=1$, in which each player has the dominant opinion.

or the bad players with low marksmanship? In this case, you have to consider the average probability of a player winning when playing against all sorts of players. Figure 4 summarizes the mathematical analysis. For the random truel and the opinion model, the histogram of winners has a maximum at a marksmanship of 100 percent. In some sense, justice is restored in this truel competition because the best players are the ones who win the tournament the most times. However, with the sequential truel rules (which favor the worst player), the distribution peaks 


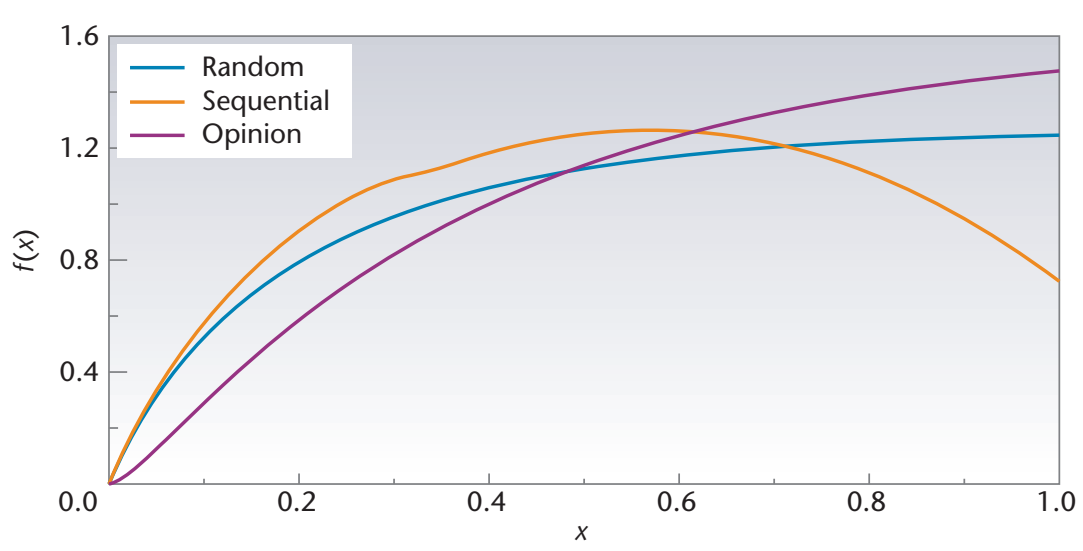

Figure 4. Distribution of winners in a truel competition. The blue line corresponds to the random truel, the orange to the sequential truel, and the purple to the convincing opinion version of the model. Note that in the sequential truel, the maximum occurs around marksmanship $x=0.55$, whereas for the other two versions, the maximum is at $x=1$.

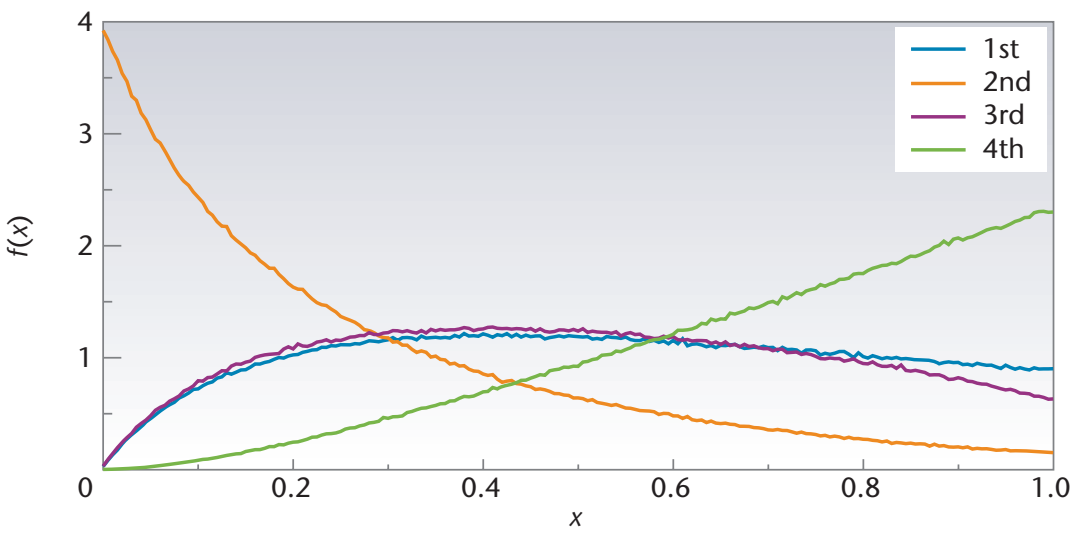

Figure 5. $\mathrm{N}$-uel. The histograms of the winner, second, third, and fourth ranked correspond to a random $\mathrm{N}$-uel tournament for $\mathrm{N}=\mathbf{4}$ players, as a function of the players' marksmanship, $x$.

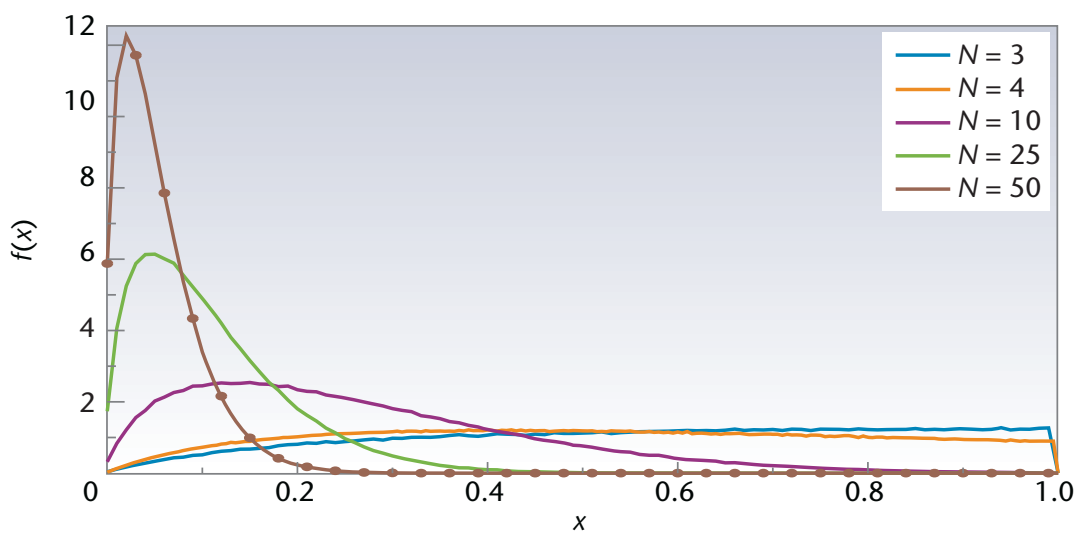

Figure 6. $\mathrm{N}$-uel. Histograms of a random $\mathrm{N}$-uel tournament's winners are a function of different values of $N$, where $N=3,4,10,25$, and 50 , as a function of the players' marksmanship, $x$. around an intermediate marksmanship of roughly 55 percent. It's the triumph of mediocrity!

\section{Generalization to $N$ Players}

If we extend the truel rules to more than three players, the situation becomes very difficult to study analytically, but it's rather easy to implement those rules in a computer program and visualize the results. Let's consider $N>$ 3 players in a random $N$-uel, but we'll still use the random truel's rules. When players use the strongest-opponent strategy, $N-1$ guns will point at the best player, so the best player's survival probability is low and, moreover, decreases with increasing $N$. In our simulations, we considered sets of $N$ players whose marksmanship we drew from a uniform random distribution in the interval $(0,1)$.

Figure 5 shows a histogram that corresponds to the classification we get when $N=4$ players are in the game. The fourth ranked corresponds to the distribution of players eliminated from the game first, the third ranked corresponds to the ones eliminated second, and so on. The distribution of the fourth ranked shows that the first individuals eliminated in the game are those with higher marksmanships. Indeed, the maximum is located at a marksmanship $x=1$, indicating that the better a player is, the higher his or her probability of being eliminated first. Another aspect we can extract from this figure deals with the distribution of the first and second ranked: these curves correspond to the case in which only two players remain in the game (that is, to a duel). Therefore, it's more likely in this situation that players with lower marksmanships are eliminated first rather than those with higher marksmanships (this is why the curve for the second ranked presents a 

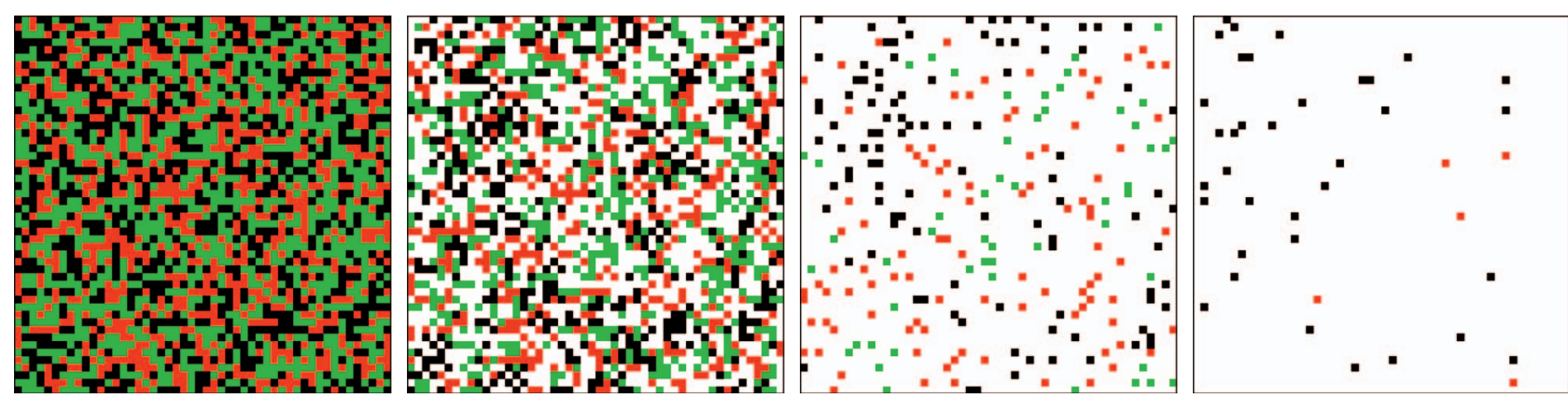

Figure 7. Simulation. Snapshots corresponding to different stages of a simulation performed for the random truel have initial proportions of $x_{A}=0.3$ (black), $x_{B}=0.3$ (red), and $x_{C}=0.4$ (green) for a set of $N=2,500$ players arranged in a twodimensional grid. The marksmanships are $a=1, b=0.8$, and $c=0.5$.

maximum in the origin). It's also worth mentioning that for four players, the histogram associated with the first ranked - that is, the winner of the 4uel-presents a maximum at a value of $x<1$. This result implies that the best performing player doesn't correspond to the player with the highest marksmanship anymore, as it did when $N=$ 3 . Indeed, the optimum value is located at roughly 0.45 .

Figure 6 shows the histogram of $N$ uel winners as a function of the number of players $N$. As in our earlier discussion, it's clear that the distribution is indeed progressively enhanced and shifted toward zero when $N$ increases. In this limiting case, we can talk about the "survival of the weak."

\section{Truels with}

\section{Spatial Dependence}

A natural step in any discussion of truels is the set of players' spatial structure. This structure reflects the fact that players don't interact with just any other player but only with those who are close in some sense. Although we could devise some sort of social network of interactions, ${ }^{20,21}$ we consider here a simple two-dimensional lattice with $N$ sites, each with four nearestneighbor links. We initialize the lattice by putting randomly on each site one player of groups $A, B$, or $C$ in the respective proportions $x_{A}, x_{B}$, and $x_{C},\left(x_{A}\right.$ $\left.+x_{B}+x_{C}=1\right)$ and respective marksmanships $a, b$, and $c$. An important part of this generalization is that players never shoot at a person of the same group. The rules of the random collective truel are as follows:

1. One of the remaining players is chosen at random.

2. The chosen player selects two players randomly from the occupied neighbors' sites, and the three of them play a random truel. The losers are eliminated from the system. If the chosen player has only one neighbor, the two will play a duel, with the loser removed from the system. If no neighbors are left, the player walks to a randomly chosen neighbor site.

3. Steps 1 and 2 repeat until all survivors belong to the same group.

In step 2, some of the chosen players could belong to the same group, and if so, they observe strictly the rule of no shooting between members of the same group. Accordingly, more than one player could survive that game. In any event, players use the strongestopponent strategy - if, for example, the three players in a truel belong to groups $A, A$, and $B$, the two $A$ players will aim at $B$, whereas $B$ will aim at one of the two $A$ s (again, chosen at random). This particular situation's outcome could be either player $B$ eliminating both $A$ players or vice versa. Because the analytical treatment seems rather difficult, let's look at the results from a direct numerical simula-

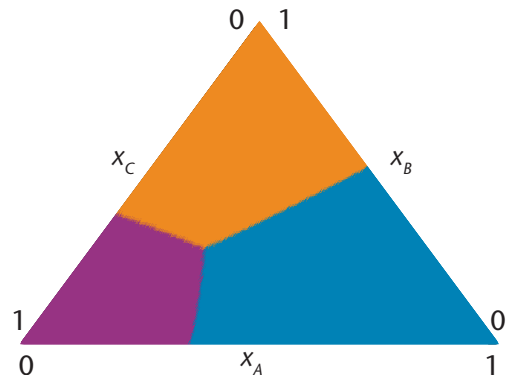

Figure 8. Random collective truel. In this diagram, the favored group-the one with the highest probability of winning (blue corresponds to group $A$, orange to $B$, and purple to $C$ ) is represented in terms of the initial proportions $x_{A}, x_{B}$, and $x_{C}$ for a set of $N=\mathbf{4 0 0}$ players arranged in a twodimensional grid. We obtained these after several simulations.

tion of the aforementioned rules. Throughout this section, we'll use the values $a=1, b=0.8$, and $c=0.5$ for the marksmanships.

Figure 7 shows snapshots at different stages of a simulation performed for the random truel. The initial population proportion was $x_{A}=0.3, x_{B}=0.3$, and $x_{C}=0.4$. Note that in the early stages of the simulation, the populations of groups $B$ and $C$ diminish, whereas group $A$ resists and eventually wins the collective truel.

In this collective truel, the group that survives at the end depends on the initial proportions of players. Figure 8 summarizes this dependence-we plot 

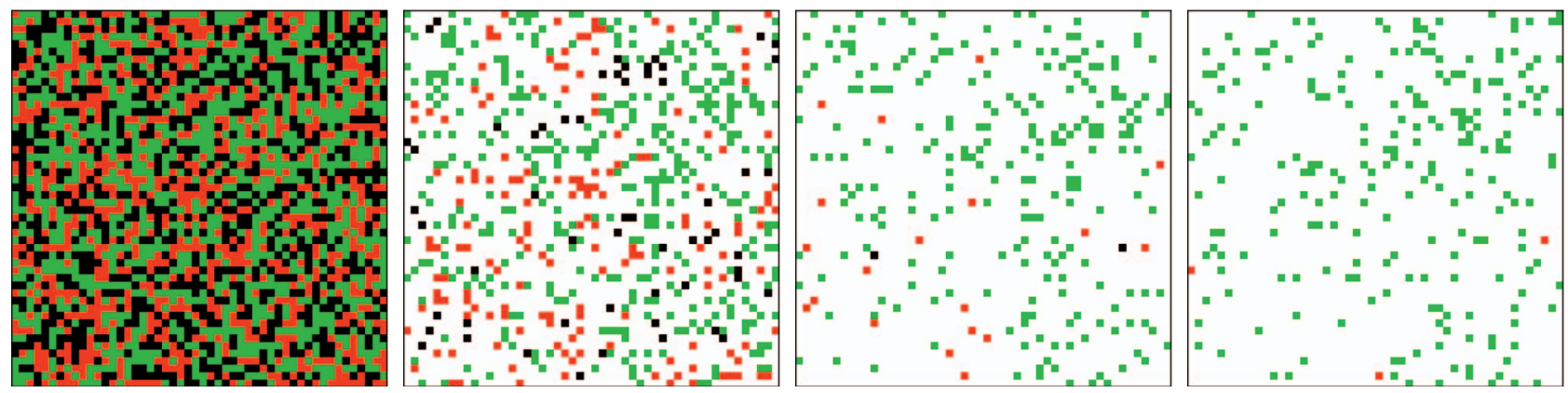

Figure 9. Sequential truel. As in Figure 8, the favored group - the one with the highest probability of winning (black corresponds to group $A$, red to $B$, and green to $C$ ) is represented in terms of the initial proportions $x_{A}, x_{B}$, and $x_{C}$ for a set of $N=400$ players arranged in a two-dimensional grid. Here, the winning group is the weakest (group $C$ ).

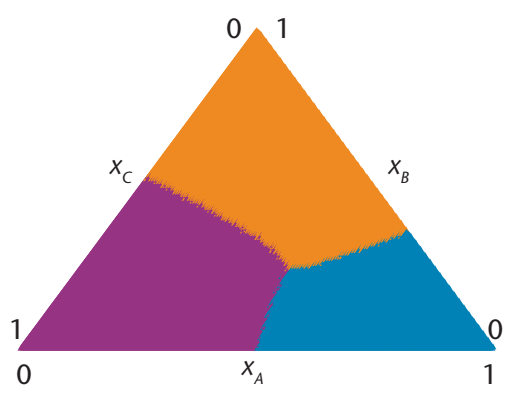

Figure 10. Sequential truel. As in Figures 8 and 9, the favored groupthe one with the highest probability of winning (blue corresponds to group $A$, orange to $B$, and purple to $C)$ is represented in terms of the initial proportions $x_{A}, x_{B}$, and $x_{C}$ for a set of $N=\mathbf{4 0 0}$ players arranged in a two-dimensional grid. Here, groups $B$ and $C$ have increased the region.

in a color code the group with the highest winning probability as a function of the initial proportions.

It's easy to modify step 2 by considering the sequential truel (players shooting in inverse order to their marksmanship). Figure 9 shows a typical realization. In this figure, the winning group is the weakest one (group $C)$; this survival of the weakest effect is also present in Figure 10, where groups $B$ and $C$ have increased the region in parameter space in which they win the truel.

Almost all truel competitions occur during the first steps, in which a large fraction of the population is removed.
At the end of this first regime, the largest remaining population is the one that possesses the higher survival probability when playing a single truel and the system has several empty sites. Later, in a second regime, players start to diffuse to neighboring sites, thus increasing the appearance of duel encounters. Consequently, the evolution will result from a balance between the population favored by the existence of duels (the one with the highest marksmanship) and the one favored by possessing a high proportion of the remaining population.

Figure 11 shows a simulation performed for the opinion model. For the set of marksmanships $a=1, b=0.8$, and $c=0.5$, we find the favorite opinion is always the one with highest marksmanship, $A$. This occurs even for very small initial proportion $x_{A}$, and it reflects the large region in the parameter space in which $A$ becomes the favorite opinion, as in Figure 3.

W e've seen that under certain circumstances, it isn't always recommendable to be the best player. Indeed, when playing the sequential truel, intermediate marksmanship values perform better on average. A next step in the model would involve a deeper study of truel dynamics in terms of the spatial grid used. Small-world or even scale-free networks introduced into the model could help us analyze the effect of different topologies on the final population. Another interesting extension for truel games would be to include dynamics based on selection and evolution. ${ }^{22}$ Strategies could evolve, in the sense that players would modify their own strategies if they contemplate the possibility of improving their own payoff.

\section{Acknowledgments}

We acknowledge financial support from the Spanish government and Fondo Europeo de Desarrollo Regional (FEDER EU) through projects FIS2004-5073 and FIS2004-953. Pau Amengual is supported by a grant from the government of the Balearic Islands.

\section{References}

1. D.M. Kilgour and S.J. Brams, "The Truel," Mathematics Magazine, vol. 70, no. 5, 1997, pp. 315-326.

2. M. Shubik, Game Theory in the Social Sciences, MIT Press, 1982.

3. M. Shubik, "Does the Fittest Necessarily Survive?" Reading in Game Theory and Political Behavior, M. Shubik, ed., Doubleday, 1954, pp. 43-46.

4. C. Kinnaird, Encyclopedia of Puzzles and Pastimes, Citadel, 1946.

5. D.M. Kilgour, "The Simultaneous Truel," Int'l J. Game Theory, vol. 1, no. 4, 1972, pp. 229-242.

6. R. Toral and P. Amengual, "Distribution of Winners in Truel Games," Modelling Cooperative Behavior in the Social Sciences, vol. 779 of AIP Conf. Proc., P.L. Garrido, J. Marro, and M.A. Munoz, eds., Am. Inst. of Physics, 2005, pp. 128-141.

7. S. Karlin, A First Course in Stochastic Processes, Academic Press, 1973. 

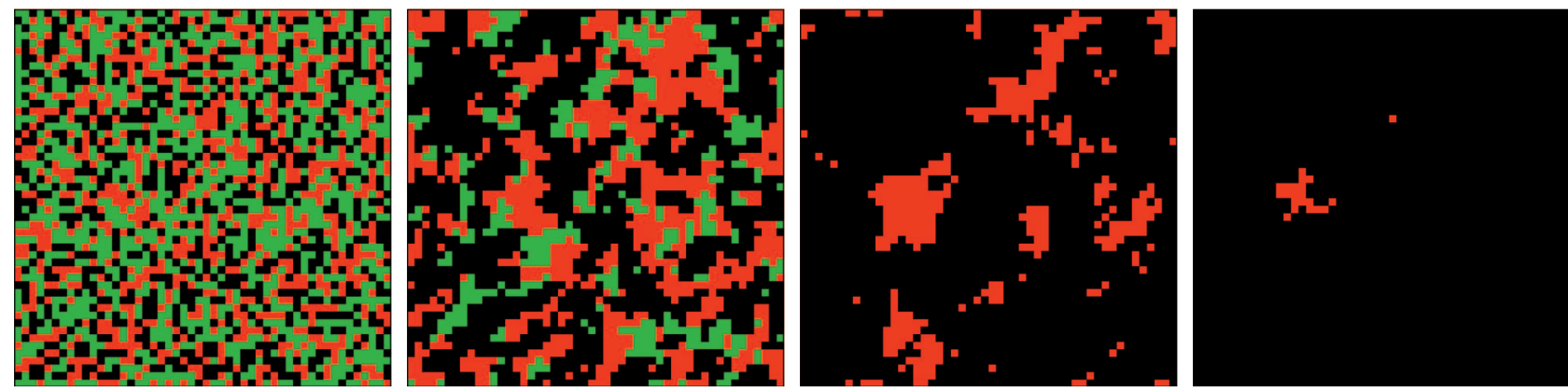

Figure 11. Opinion truel. Snapshots corresponding to different stages of a simulation performed for the opinion truel have initial proportions of $x_{A}=0.3$ (black), $x_{B}=0.3$ (red), and $x_{C}=0.4$ (green) for a set of $N=2,500$ players arranged in a two-dimensional grid. The marksmanships are $a=1, b=0.8$, and $c=0.5$.

8. G.P. Harmer and D. Abbott, "A Review of Parrondo's Paradox," Fluctuation and Noise Letters, vol. 2, no. 2, 2002, pp. R71-R107.

9. M.V. Smoluchowski, "Experimentell nachweishare, der ublichen thermodynamik widersprechende molekilarphanomene" [in German], Physikalische Zeitschrift, vol. 13, 1912, p. 1069.

10. R.P. Feynman, R.B. Leighton, and M. Sands, The Feynman Lectures on Physics, AddisonWesley, 1963.

11. J.M.R. Parrondo and P. Espanol, "Criticism of Feynman's Analysis of the Ratchet as an Engine," Am. J. Physics, vol. 64, no. 9, 1996, pp. 1125-1130.

12. B. Sinervo and C.M.M. Lively, "The RockScissors-Paper Game and the Evolution of Alternative Male Strategies," Nature, vol. 380, no. 6571, 1996, pp. 240-243.

13. M. Frean and E.R. Abraham, "Rock-ScissorsPaper and the Survival of the Weakest," Proc. Royal Soc. London B, vol. 268, no. 1474, 2001, pp. 1323-1327.

14. K. Tainaka, "Paradoxical Effect in a ThreeCandidate Voter Model," Physics Letters A, vol. 176, no. 5, 1993, pp. 303-306.

15. D.M. Kilgour, "The Sequential Truel," Int'll. Game Theory, vol. 4, no. 3, 1975, pp. 151-174.

16. D.M. Kilgour, "Equilibrium Points of Infinite Sequential Truels," Int'l J. Game Theory, vol. 6, no. 3, 1977, pp. 167-180.

17. W. Bossert, S.J. Brams, and D.M. Kilgour, "Cooperative vs. Non-Cooperative Truels: Little Agreement, but Does that Matter?" Games and Economic Behavior, vol. 40, no. 2, 2002, pp. 185-202.

18. A.P. Flitney and D. Abbott, "Quantum Twoand Three-Person Duels," J. Optics B: Quantum and Semiclassical Optics, vol. 6, no. 8, 2004, pp. S860-S866.

19. M.J. Osborne and A. Rubinstein, A Course in Game Theory, MIT Press, 1997.

20. D.J. Watts and S.H. Strogatz, "Collective Dynamics of Small-World Networks," Nature, vol. 393, no. 6684, 1998, pp. 440-442.

21. S.N. Dorogovtsev and J.F.F. Mendes, "Evolution of Networks," Advances in Physics, vol.
51, no. 4, 2002, pp. 1079-1187.

22. J. Hofbauer and K. Sigmund, Evolutionary Games and Population Dynamics, Cambridge Univ. Press, 1998.

Pau Amengual is a PhD student at the University of the Balearic Islands. His technical interests include statistical physics, Brownian motors, and Parrondo's games. Amengual has a BS in physics from the University of the Balearic Islands. Contact him at pau@

\section{THE IEEE'S 1ST ONLINE-ONLY MAGAZINE}

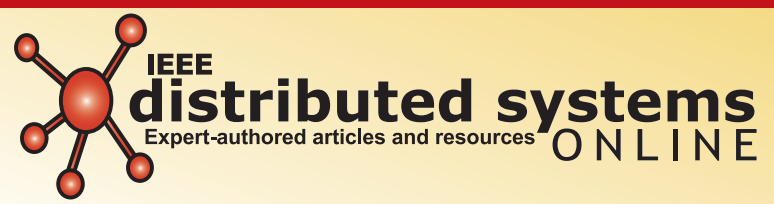

\section{IEEE Distributed Systems Online brings you}

peer-reviewed articles, detailed tutorials, expert-managed topic areas, and diverse departments covering the latest news and developments in this fast-growing field.

\section{Log on for free access}

to such topic areas as

$$
\begin{gathered}
\text { Grid Computing • Middleware } \\
\text { Cluster Computing • Security } \\
\text { Peer-to-Peer • Operating Systems } \\
\text { Web Systems • Parallel Processing } \\
\text { Mobile \& Pervasive } \\
\text { and More! }
\end{gathered}
$$

To receive monthly updates, email dsonline@computer.org

$$
\text { http://dsonline.computer.org }
$$

